\title{
Variations
}

Variations

Revue internationale de théorie critique

11 | 2008

La beauté est dans la rue

\section{« La lutte continue »}

Réassurer Clichy-sous-Bois pour que ne se désespère plus Billancourt

\section{Stéphane Le Lay}

\section{(2) OpenEdition}

Journals

\section{Édition électronique}

URL : http://journals.openedition.org/variations/258

DOI : $10.4000 /$ variations. 258

ISSN : 1968-3960

Éditeur

Les amis de Variations

\section{Référence électronique}

Stéphane Le Lay, " «La lutte continue » », Variations [En ligne], 11 | 2008, mis en ligne le 01 février 2012, consulté le 02 mai 2019. URL : http://journals.openedition.org/variations/258 ; DOI : 10.4000/ variations. 258

Ce document a été généré automatiquement le 2 mai 2019.

Les ami•e•s de Variations 


\title{
« La lutte continue »
}

\author{
Réassurer Clichy-sous-Bois pour que ne se désespère plus Billancourt
}

\section{Stéphane Le Lay}

\section{NOTE DE L'ÉDITEUR}

Première publication sur www.theoriecritique.com, « La beauté est dans la rue », 1er mai 2008, pp. 28-33

1 Mai 1968 semble être sur toutes les lèvres et dans tous les esprits ces derniers jours. On ne compte plus les sorties d'ouvrages sur la question, plus ou moins opportunistes ${ }^{1}$. La tonalité des débats oscille généralement entre deux extrêmes: d'un côté, la nostalgie d'une époque où l'espoir portait la puissance de devenirs meilleurs, car moins compassés et rigides; de l'autre, la reconnaissance d'un ensemble de phénomènes à l'existence historique indéniable, mais désormais révolue voire à liquider².

2 Entre les deux, tentant de décrire au plus près les centaines de micro-événements survenus dans cette configuration sociopolitique particulière, ou de rendre compte des luttes interprétatives qui s'ensuivirent, émergent des analyses plus fines. Ainsi, certaines insistent par exemple sur l'inscription internationale du fait national, puisque, par-delà des différences évidentes, des luttes contre les formes d'oppression bureaucratique et l'exploitation capitaliste des énergies productives se sont produites aussi bien en Italie, qu'au Mexique ou en Tchécoslovaquie. À l'inverse, d'autres analyses se concentrent sur le cas français, pour décrire minutieusement l'ancrage provincial et populaire du conflit, plus tard hypostasié en un mouvement étudiant parisien par certains intellectuels, moins soucieux de réalisme historique que de stratégie politique personnelle (réduire un mouvement d'ensemble, parfois contradictoire, à sa partie «bourgeoise» pour en vilipender - ou au contraire s'en féliciter - les impasses individualistes).

3 Écouter d'autres voix revenir sur le contexte, les formes multiples de déploiement et la résolution socioéconomique et politique du «joli mois de mai » a de multiples intérêts. L'un d'eux, et non des moindres, est de pouvoir effleurer, à défaut d'en embrasser une compréhension complète, la diversité des enjeux engagés dans l'action (ou au contraire 
dans la fuite) : enjeux politiques (avec ses multiples dissensions, ses espoirs, ses craintes, etc.), enjeux économiques (comment les structures capitalistes ont-elles encaissé le choc d'une remise en cause si brutale de leurs organisation et finalités?), mais également enjeux pulsionnels (que signifiait donc cette demande éruptive de parole, sans nécessairement chercher à conquérir le pouvoir ? Comment la violence des affrontements pouvait-elle si facilement côtoyer la légèreté et l'humour de certaines situations ?). Se reposer précisément ces questions garde, me semble-t-il, toute sa pertinence.

Un autre intérêt de ne pas bouder les débats sur mai 1968, tout autant crucial, est de pouvoir se rappeler que, pendant le travail d'inventaire, des luttes perdurent, qui parfois résonnent comme un écho à des préoccupations anciennes. D'où une question que l'on peut légitimement se poser: quarante ans après avoir fait bouger certaines lignes de rigidité de son temps, le conflit collectif de mai 1968 saura-t-il donner des idées à la génération suivante?

\section{Deux configurations sociales bien distinctes}

5 Faut-il le souligner?, la vie en 2008 n'a pas grand-chose à voir avec celle de 1968. Quand les unes et les autres contestaient une société d'autant plus étouffante qu'étaient entrevus des changements sociaux profonds et positifs (rapport au corps, rapport au temps, etc.), les générations nées à partir des années 1970 se trouvent confrontées à une fermeture des possibles particulièrement anxiogène. Avec le recul, mai 1968 peut en effet encore apparaître comme un moment où les forces progressistes réussiraient à faire reculer les injustices les plus insupportables (améliorations des conditions de vie des classes populaires, mouvements féministes, mouvements des droits civiques et lutte contre la guerre du Viêt-nam aux États-Unis, etc.), en dépit de certains signes annonciateurs d'une reprise en main conservatrice qui ne tarderait plus (assassinat de Martin Luther King, écrasement du «printemps de Prague» par les forces du bloc soviétique entre autres exemples). En France, même si la crainte du chômage commençait à prendre forme dans les esprits, l'heure était davantage à l'offensive ouvrière qu'à son affaiblissement.

En 2008, précarisation sociale et répression des "désordres» de toutes natures constituent le lot quotidien de millions de personnes se vivant parfois difficilement comme des individus différents œuvrant à une communauté de semblables ${ }^{3}$. L'effet de fragmentation qui s'ensuit n'a alors rien de commun avec l'utopie portée par les manifestants de mai, même si l'on n'oublie pas l'éparpillement politique qui prévalait lors des " événements" ${ }^{4}$. Difficile en effet au « rire politique » de mobiliser à partir de mots d'ordre optimistes, quand tout indique que les situations se détériorent, et que le mouvement s'amplifie de jour en jour. Ceci est d'autant plus vrai qu'une partie des élites économiques, politiques et médiatiques n'a eu de cesse, ces trente dernières années, d'opposer des catégories de populations à d'autres, dans le but d'accentuer la division de ce qui constituait auparavant les membres des forces progressistes du pays ${ }^{5}$.

7 Nous nous trouvons ainsi face à une configuration dont les dynamiques structurantes sont particulièrement difficiles à modifier : d'un côté, une bourgeoisie à la puissance reconstituée ${ }^{6}$, dont une partie rien moins que négligeable n'a que faire des difficultés quotidiennes de la grande majorité de la population, et se consacre à son entre-soi ${ }^{7}$; certes, une strate bourgeoise plus ouverte et moins fortunée existe, mais elle se comporte bien souvent de manière condescendante avec les milieux populaires, comportement 
produisant des effets de démobilisation propres ${ }^{8}$. La polarisation de cette puissance se donne à voir dans tous les domaines de la vie humaine, cumul entraînant le renforcement des positions dominantes?.

8 D'un autre côté, un "archipel salarié » à la topographie déchirée ${ }^{10}$ selon l'état d'avancement de la déstructuration des principes de cohésion propres à l'État social, auquel aucun niveau d'intégration supérieur (l'Europe notamment) ou inférieur (la Région, par exemple) n'a réussi (tenté ?) à se substituer.

Si subsistent des lieux protégés dans les différentes fonctions publiques, en dépit d'une dégradation des conditions d'emploi et de travail, principalement dans les catégories les plus basses ${ }^{11}$, le secteur privé connaît maintenant depuis une vingtaine d'années une recrudescence spectaculaire de l'exploitation et de l'oppression. L'industrie fut la première à payer le prix des nouvelles orientations stratégiques, et ce quelques années à peine après la dernière grande grève ouvrière (mai 1968). Pendant ce temps-là, le monde des luttes du tertiaire (notamment du secteur public) se faisait discret: traditions incompatibles? structurations différentes? politique du dos rond? divisions syndicales? Sans doute ces raisons ont-elles influé sur l'incapacité à créer des fronts unifiés de mouvements sociaux pour proposer (imposer ?) des alternatives à la destruction planifiée de régions entières.

10 Une fois obtenue la " défaite de la classe ouvrière », il devenait facile pour les « nouveaux réformateurs " d'intensifier les attaques en direction des autres secteurs, en réutilisant parfois les mêmes méthodes : dorénavant, les fonctions publiques se trouvent fortement stratifiées selon les statuts d'emploi. Quant au secteur tertiaire privé, l'usage des béances du Code du travail au sein d'entreprises de taille réduite permet d'organiser la production sur une division du travail fortement individualisée (la qualification devenue compétence fonctionnant à géométrie variable selon le sexe, l'âge, la race et les capacités de négociation des individus recrutés) ${ }^{12}$.

11 Tableau apocalyptique penseront certains. Bien loin de la réalité, répondrai-je. Car c'est sans compter les chômeurs, les incarcérés pour cause économique, les retraités faiblement pensionnés, etc. C'est également loin d'exprimer les drames humains qui se jouent dans les corps et les esprits, pris au sein des processus brossés ici à larges traits.

\section{Absence de conflits et confettis de luttes : comment rallumer le feu?}

12 Derrière cette souffrance indéniable aux effets multiformes, il faudrait être cependant de bien mauvaise foi, ou singulièrement myope, pour affirmer que la conflictualité sociale a disparu. Peu s'y aventurent depuis quelques mois. Les phénomènes de grève et de manifestation constituent toujours des moyens disponibles dans l'arsenal militant. Et si leur présence fluctue selon la conjoncture économique et politique, on ne saurait les remiser d'un revers de la main au musée des vieilleries ${ }^{13}$. Mais on doit également prendre le temps de distinguer les mouvements porteurs de positivité de ceux destinés à exprimer la colère, la frustration, le désespoir. Leurs formes et leurs messages diffèrent radicalement, tout comme, bien souvent, leur issue. Force est de constater que, depuis une vingtaine d'années, les conflits relatifs aux questions du travail et de l'emploi se sont généralement caractérisés par des actes peu créatifs, défensifs et aux effets sans grande portée. Ces limites rappelées, on peut malgré tout remarquer que les révoltes de 
novembre 2005 et le mouvement anti-CPE de 2006, au-delà de leurs différences sociologiques et événementielles, ont constitué deux réponses à la disciplinarisation croissante de la société (ce qui n'est pas sans évoquer les mouvements de mai 1968), qu'on l'envisage dans sa dimension économique ou politique (vie de la cité).

13 L'ébranlement provoqué par ces deux mouvements n'est sans doute pas pour rien dans la capacité de la droite radicale à remporter les échéances électorales de 2007, se présentant comme la seule force apte à lutter contre le dépérissement de l'autorité étatique causé par les « ennemis intérieurs » (les « parasites » de l'assistance sociale, les « fainéants » des $35 \mathrm{~h}$, etc.), et à moderniser le pays pour affronter les enjeux actuels. Une large partie du monde salarial a entendu ce message, et l'a pris au pied de la lettre, peut-être insuffisamment assurée de ses propres capacités à peser sur le jeu politique sans en passer par le recours à une figure providentielle maniant la rhétorique avec efficacité ${ }^{14}$.

14 Car notre économie se compose d'un secteur tertiaire extrêmement développé, impliquant une territorialisation importante de l'activité (en dépit de la possibilité de délocaliser quelques activités emblématiques comme les call center). Dans cette configuration, les salariés bénéficient d'un avantage qu'il serait idiot de ne pas chercher à mettre à profit; pour cela, il faut réassurer les membres des classes populaires du potentiel de leur puissance sociale, des possibilités existantes dans le remodelage du réel (ici le travail des intellectuels peut servir, en fournissant des données économiques et sociales visant à montrer que les richesses à redistribuer ou créer différemment existent bien encore dans les territoires locaux et qu'elles ne sont pas uniquement créées ex nihilo par les forces spéculatives des marchés financiers).

15 Certes, les forces syndicales, largement affaiblies ${ }^{15}$, ne peuvent guère investir les myriades d'entreprises, aux contraintes variées, qui emploient des individus souvent peu au fait du droit social. Mais ces forces ne sont pas sans atouts. Notamment, elles sont ancrées dans certains bastions publics encore relativement protégés. On pourrait alors envisager une forme croisée de parrainage intersectoriel sur base territoriale: par exemple, un fonctionnaire serait chargé de faire le lien avec un salarié d'une entreprise donnée (au hasard, les caissières d'une grande surface), de manière à pouvoir faire intervenir des forces syndicales implantées, en cas de difficultés particulières, ou pour appuyer une revendication sectorielle. De la même manière, lors de l'occupation d'une école, on pourrait envisager que des salariés du privé viennent soutenir les enseignants, comme cela se fait déjà avec certains parents d'élèves.

Si je prends les cas de l'école et des caissières de supermarché, la raison n'en est pas totalement fortuite. D'abord, ces deux professions sont fortement féminisées. Or, dans les luttes, planent parfois sur les femmes des contraintes d'un type particulier qu'il conviendrait de faire diminuer (violence, déni de légitimité, etc.) ${ }^{16}$. En croisant les secteurs d'activité et en mettant ainsi en présence des individus évoluant dans des univers sociaux différents, certains des freins pourraient en partie être levés. Ensuite, on peut envisager que les enfants constituent un point d'articulation facilitant les contacts, notamment dans des quartiers touchés par des formes diverses de discriminations. Enfin, école et commerces (alimentaires ou non) sont des lieux importants, symboliquement et matériellement. En organiser des liens de combativité permettrait d'expérimenter une forme d'action visible dans une ville (du moins dans sa partie la plus périphérique dans un premier temps), et porteuse d'exemplarité (les bataillons d'enseignants et de salariés du commerce pourraient en effet se répartir de manière à toucher d'autres secteurs d'activité du territoire - je pense notamment aux secteurs de la coiffure et de la 
restauration, friands d'apprentis malléables ou de main-d'œuvre immigré clandestine, et par ailleurs excessivement rentables).

Des victoires dans l'organisation du travail et les salaires au niveau local auraient plus qu'une portée symbolique. Elles pourraient démontrer que la tendance actuelle au détricotage des droits sociaux et à la baisse continue des salaires peut trouver une voie de résolution différente (certains régimes spéciaux toujours actifs, notamment en Alsace, sont là pour le rappeler). Une voie qui passe par la mise en action des puissances localisées, comme en leur temps le furent les conflits du « joli mois de mai ».

\section{NOTES}

1. Un encadré inséré à l'article de Jean Birnbaum consacré à la question, dans le Monde des livres, dénombre, au 20 mars 2008, 80 livres (http://www.lemonde.fr/livres/article/2008/03/20/mai-68en-heritage_1025308_3260.html?xtor=RSS-3260).

2. Je fais l'hypothèse qu'est connu le discours de Sarkozy à ce propos, prononcé lors de la campagne présidentielle de 2007. Le lecteur intéressé par une mise en perspective historique de l'origine intellectuelle des différentes positions anti-soixante-huitardes pourra se reporter à l'ouvrage de S. Audier, La pensée anti-68. essai sur les origines d'une restauration intellectuelle, La Découverte, 2008. Dès l'introduction, l'auteur souligne que «les chefs d'accusation sont assez répétitifs : nihilisme, individualisme, narcissisme, relativisme, postmodernisme, égalitarisme niveleur, débauche hédoniste, "jeunisme", destruction de l'autorité, discrédit de la "valeur travail", perte du sens de la hiérarchie, mépris pour l'“excellence", ruine de la citoyenneté, mort de la culture, destruction de l'école, ruine de la nation, etc. Surtout, à droite comme à gauche, on voit souvent réapparaître la thèse paradoxale que les acteurs de mai 1968 ont joué un rôle clé dans le déploiement du capitalisme de la fin des années 1970, en faisant sauter le dernier verrou qui limitait le plein essor de la marchandisation du monde : celui des mœurs traditionnelles. De ce point de vue, la droite traditionaliste peut rejoindre certains segments de la gauche intellectuelle dans le procès de 68 , accusé d'avoir détruit les fondations mêmes de la société.» (p.21)

3. J'ai essayé de montrer ailleurs que, chez certains membres des classes populaires, existent des processus de contre-stigmate qui, tout en permettant de lutter contre la souffrance sociale liée au déclassement et au mépris sociaux, ont tendance à opposer les individus entre eux selon des catégories de perception fortement naturalisées. S. Le Lay, Autonomie individuelle et précarisation. Dispositifs publics et souffrance sociale en classes populaires, Thèse de sociologie (2 tomes), Université Paris VIII, Saint-Denis, 2004.

4. Pour une illustration "à chaud» de cette question au niveau étudiant, lire par exemple Mouvement du 22 mars, Ce n'est qu'un début continuons le combat, La Découverte, 2001. On peut suivre, presque pas à pas, la manière dont un groupe connu sous le nom des «enragés de Nanterre " s'est engagé dans la lutte contre « les forces de répression policière et l'organisation universitaire ». Les dissensions internes et externes, selon les lignes de partage politiques, y apparaissent clairement. Pour une vision plus analytique et distanciée, se reporter à B. Gobille, Mai 68, La Découverte (Repères), 2008. L'auteur rappelle notamment les relations difficiles entre les mouvements d'étudiants et la CGT, qui ont contribué à freiner la jonction entre grèves 
étudiantes et ouvrières, sans pour autant les rendre impossibles dans de nombreuses villes de province.

5. S. Le Lay, «La droite et le mouvement social du printemps 2003 : amorce d'une lutte anthropologique? », Mouvements, 2004, n³5, pp.33-41.

6. T. Piketty, Les hauts revenus en France au Xxe siècle. Inégalités et redistributions, 1901-1998, Grasset \& Fasquelle, Paris, 2001.

7. M. Pinçon et M. Pinçon-Charlot, Sociologie de la bourgeoisie, La Découverte \& Syros (Repères), Paris, 2000.

8. En témoigne l'éloignement des classes populaires avec les appareils et les instances politiques, aussi bien représentatives que participatives.

9. Pour un panorama précis du système des inégalités (envisagées sous leur angle classiste - sans toutefois méconnaître leurs dynamiques sexuées et ethniques ou raciales), voir A. Bihr et $\mathrm{R}$. Pfefferkorn, Le système des inégalités, La Découverte (Repères), 2008.

10. P. Cours-Salies et S. Le Lay (dir.) Le bas de l'échelle. Constructions sociales des situations subalternes , Ramonville Saint-Agne, Erès, 2006.

11. C. César, «L'Éducation nationale et les ouvrier(e)s : le mammouth et les souris ? ", in P. CoursSalies et S. Le Lay (dir.) Le bas de l'échelle..., op. cit., pp.97-107.

12. Pour les derniers développements en matière d'attaques contre le droit du Travail, se reporter à G. Filoche, «Tornade patronale sur le code du travail », Le monde diplomatique, mars 2008, pp.12-13.

13. A. Carlier et É. Tenret, «Des conflits du travail plus nombreux et plus diversifiés », Premières synthèses, $\mathrm{n}^{\circ} 08.1,2007$.

14. S. Le Lay, «La politique a-t-elle vocation à être de droite? Contribution au débat dans la gauche ", Nouvelles fondations, $\mathrm{n}^{\circ} 7,2007, \mathrm{pp} .8-13$.

15. J.-M. Pernot, Syndicats : lendemains de crise?, Gallimard (Folio), Paris, 2005.

16. S. Le Lay, «Une disposition au désengagement militant : processus de précarisation salariale et démobilisation raisonnée ", in P. Cardon, D. Kergoat et R. Pfefferkorn (dir.), La dialectique de l'individuel et du collectif dans les rapports sociaux de sexe (titre provisoire), La Dispute (Le genre du monde), à paraître fin 2008.

\section{AUTEUR}

\section{STÉPHANE LE LAY}

Sociologue 